\title{
REPRESENTAÇÃO DOS ESTADOS DE ACOPLAMENTO ATMOSFÉRICO ATRAVÉS DE UM MODELO DE COLUNA SIMPLES
}

\author{
Moacir Schmengler ${ }^{1, *}$, Felipe Denardin Costa $^{2}$, Otávio Costa Acevedo ${ }^{3}$ \\ ${ }^{1}$ Centro de Previsão de Tempo e Estudos Climáticos, INPE, Cachoeira Paulista, SP, Brasil \\ ${ }^{2}$ Universidade Federal do Pampa, Campus Alegrete, Alegrete, RS, Brasil \\ ${ }^{3}$ Universidade Federal de Santa Maria, Santa Maria, RS - Brasil \\ *moacir.schmengler@cptec.inpe.br
}

\section{RESUMO}

É utilizado um Modelo de Coluna Simples com o propósito de reproduzir os estados de acoplamento atmosférico na Camada Limite Planetária em condições muito estáveis. O modelo é composto por equações prognósticas para algumas componentes médias e por uma equação para o balanço de energia em superfície. Verifica-se que os esquemas são capazes de reproduzir o processo de acoplamento, contudo apenas para valores muito altos de vento geostrófico.

\section{ABSTRACT}

A Single Column Model is used in order to reproduce the coupling states of a Planetary Boundary Layer under very stable conditions. The model consists of prognostic equations for some mean components and for the surface energy budget. It is found that the schemes are able to reproduce the coupling process, but only for very high values of geostrophic wind.

\section{INTRODUÇÃO}

A representação matemática dos processos físicos que ocorrem no interior da Camada Limite Planetária (CLP) tem grande impacto em inúmeras questões associadas à Meteorologia. 
Assim, com o propósito de melhorar a representação dos processos verticais e unidimensionais por natureza e dos processos de interação entre a superfície e a atmosfera, vários estudos consideram os chamados Modelos de Coluna Simples (MCS). Os MCS consistem em uma grade vertical unidimensional, na qual são resolvidas as quantidades que governam o escoamento turbulento. Contudo, trabalhos anteriores mostraram que este tipo de modelo apresenta enorme dificuldade em reproduzir os fenômenos de conexão ou de desconexão que ocorrem durante a noite, quando a Camada Limite apresenta um perfil muito estável (CLE). Assim, neste trabalho será utilizado um Modelo de Coluna Simples com o propósito de reproduzir estes fenômenos. Para isso serão utilizadas quatro diferentes formulações turbulentas e uma equação prognóstica para a temperatura da superfície do solo, baseada no balanço de energia junto à superfície. Para isto foram utilizadas as condições iniciais observadas na noite que se iniciou em 28 de julho de 2001, obtidas em um sítio experimental localizado próximo a cidade de Santarém - PA.

\section{MODELO}

Sendo a CLE não subsidente, horizontalmente homogênea e sem divergência do fluxo radioativo, as equações prognósticas para as componentes médias do vento $\bar{u}$ e $\bar{v}$ para a temperatura potencial $(\bar{\theta})$ e para a energia cinética turbulenta $\bar{e}$ podem ser escritas como:

$$
\begin{gathered}
\frac{\partial \bar{u}}{\partial t}=f\left(\bar{v}-\bar{v}_{G}\right)-\frac{\partial}{\partial z}\left(\overline{u^{\prime} w^{\prime}}\right) \\
\frac{\partial \bar{v}}{\partial t}=f\left(\bar{u}_{G}-\bar{u}\right)-\frac{\partial}{\partial z}\left(\overline{v^{\prime} w^{\prime}}\right) \\
\frac{\partial \bar{\theta}}{\partial t}=-\frac{\partial}{\partial z}\left(\overline{w^{\prime} \theta^{\prime}}\right) \\
\frac{\partial \bar{e}}{\partial t}=-\overline{u^{\prime} w^{\prime}} \frac{\partial \bar{u}}{\partial z}-\overline{v^{\prime} w^{\prime}} \frac{\partial \bar{v}}{\partial z}+\frac{g}{\Theta}\left(\overline{w^{\prime} \theta^{\prime}}\right)-\frac{\partial}{\partial z}\left(\overline{w^{\prime} e}\right)-c_{\varepsilon} \frac{\bar{e}^{3 / 2}}{l_{d}}
\end{gathered}
$$


onde $\bar{u}_{G}$ e $\bar{v}_{G}$ são as componentes do vento geostrófico, $f$ é o parâmetro de Coriolis, $\left(\overline{u^{\prime} w^{\prime}}\right)$

e $\left(\overline{v^{\prime} w^{\prime}}\right)$ são as componentes do fluxo de momento, $\left(\overline{\theta^{\prime} w^{\prime}}\right)$ é o fluxo de calor sensível, $\left(\overline{w^{\prime} e}\right)$ é o fluxo turbulento de energia cinética, $g$ é a aceleração da gravidade, $\Theta$ é a temperatura de referência, $c_{\varepsilon}$ é uma constante e $l_{d}$ é o comprimento de mistura associado a dissipação viscosa de turbulência.

Os fluxos turbulentos foram parametrizados através dos respectivos coeficientes de difusão, utilizando-se quatro diferentes formulações para representar o comprimento de mistura (ver Tabela 1).

Tabela 1: Diferentes formulações de $l_{m}$ utilizadas nas simulações.

\begin{tabular}{l|l}
\hline Parametrização & Comprimentos de mistura \\
\hline Delage (1974) -D74 & $\frac{1}{l_{m}}=\frac{1}{\kappa\left(z+z_{0}\right)}+\frac{1}{\ell_{0}}+\frac{\beta_{c}}{\kappa L}$ \\
Louis (1979) -L79 & $l_{m}=\min (\kappa z, 200) f_{m} ; \quad f_{m}=(1+4.7 R i)^{-2}$ \\
Delage (1997) -D97 & $l_{m}=\min (\kappa z, 200) f_{m} ; \quad f_{m}=(1+12 R i)^{-2}$ \\
Belair et al.(1999) -B99 & $l_{m}=\min (\kappa z, 200) f_{m} ; \quad f_{m}=(1+12 R i)^{-1}$ \\
\hline
\end{tabular}

Para fazer as análises do comportamento das variáveis médias que controlam o escoamento atmosférico na CLP noturna foi considerada uma fina camada de vegetação que interage com o solo e a atmosfera. No interior desta camada de vegetação, o calor é distribuído uniformemente, fazendo com que a temperatura de toda a camada vegetada seja 
praticamente a mesma da temperatura da vegetação em superfície. Assim, a equação de balanço de energia na superfície vegetada pode ser escrita como:

$$
C_{v} \frac{d T_{v e g}}{d t}=Q_{n e t}-G_{0}-H_{0}-L_{v} E
$$

onde $C_{v}$ é a capacidade calorífica da camada de vegetação, $T_{v e g}$ é a temperatura da vegetação, $Q_{n e t}$ é o balanço radiativo na superfície vegetada, $G_{0}$ é o fluxo de calor no solo, $H_{0}$ é o fluxo de calor sensível em superfície e $L_{v} E$ é o fluxo de calor latente em superfície. O balanço radiativo em superfície é dado por $Q_{n e t}=\varepsilon_{a} \sigma_{s} T_{a}^{4}+60 \cdot N-\varepsilon_{s} \sigma_{s} T_{v e g}^{4}$ (van de Wiel et al. 2002).

\section{RESULTADOS E DISCUSSÃO}

Figura 1 mostra a dependência detalhada da estrutura térmica em termos do forçante mecânico $\left(\mathrm{u}_{\mathrm{G}}\right)$ no topo da CLE. O estado de conexão é representado por um rápido aumento das temperaturas nos níveis mais baixos a partir de um valor específico de $\mathrm{u}_{\mathrm{G}}$. Isto ocorre em torno de $\mathrm{u}_{\mathrm{G}}=35 \mathrm{~m} \mathrm{~s}^{-1}$ para a formulação $\mathrm{D} 74, \mathrm{u}_{\mathrm{G}}=25 \mathrm{~m} \mathrm{~s}^{-1}$ para D97 e $\mathrm{u}_{\mathrm{G}}=16 \mathrm{~m} \mathrm{~s}^{-1}$ para L79 e B99.

Entretanto, é importante destacar que a transição entre os regimes desconectado e conectado é bem menos abrupta que mostrado, por exemplo, por McNider et al. (1995) e por Acevedo et al. (2012). Evidências experimentais sobre esse aspecto indicam que a transição é abrupta e ocorre em valores de vento acima da CLE entre 3 a $7 \mathrm{~m} \mathrm{~s}^{-1}$ (Acevedo e Fitzjarrald, 2003; van de Wiel et al., 2012). Este fato mostra a ineficiência dos Modelos de Coluna Simples tem em reproduzir o estado de conexão entre a superfície e os níveis mais elevados da CLE. 

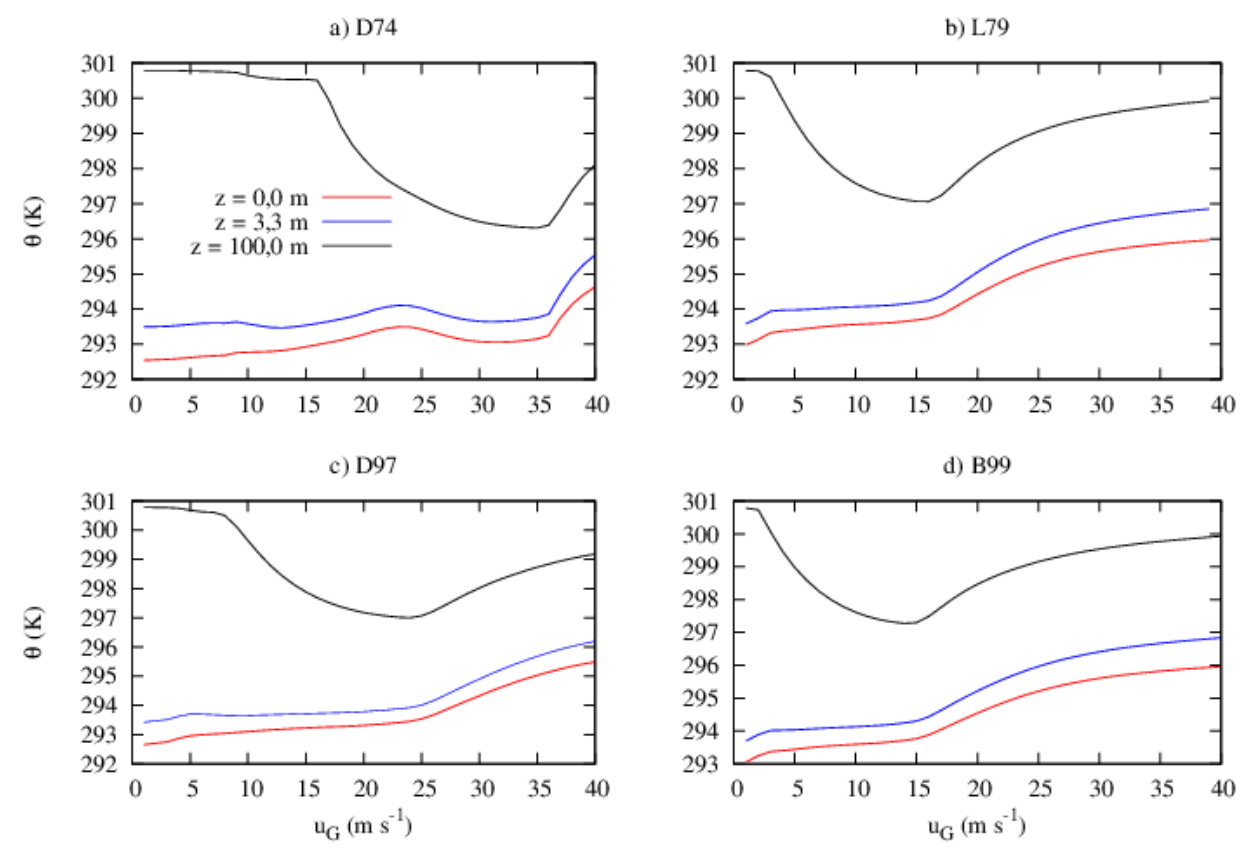

Figura 1: Temperatura potencial em função do vento geostrófico para diferentes níveis verticais (indicados pela legenda) e para diferentes parametrizações de turbulência (indicados pelo título de cada painel).

\section{CONSIDERAÇÕES FINAIS}

De fato, trabalhos anteriores nunca mostraram que os Modelos de Coluna Simples fossem capazes de reproduzir este comportamento e é este o motivo pelo qual no presente trabalho as análises foram feitas até limites irrealisticamente grandes de $\mathrm{u}_{\mathrm{G}}$.

É interessante verificar que os esquemas utilizados neste trabalho até são capazes de reproduzir este processo, contudo ele apenas ocorre para valores que não ocorrem de fato no mundo real. A conexão ocorre apenas quando todo o domínio vertical está turbulento. Os MCS têm, tipicamente, um domínio grande que pode chegar a alguns quilômetros, fazendo com que esta situação somente seja obtida com ventos excessivamente intensos. Por outro lado, em modelos simplificados de poucas camadas, a transição tipicamente ocorre em valores mais realísticos, de 3 a $12 \mathrm{~m} \mathrm{~s}^{-1}$ (MCNIDER et al., 1995; van de WIEL et al., 2002; ACEVEDO et al.,2012). 


\section{REFERÊNCIAS}

ACEVEDO, O. C.; COSTA, F. D.; DEGRAZIA, G. A. Turbulence formulation influence on the coupling state of an idealized stable boundary layer. Boundary-Layer Meteorology, v. 145, p. 211-228, 2012.

ACEVEDO, O. C.; FITZJARRALD, D. In the core of the night-effects of intermittent mixing on a horizontally heterogeneous surface. Boundary-Layer Meteorology, v. 106, p. $1-33,2003$.

BELAIR, S.; MAILHOT, J.; STRAPP, J. W.; MACPHERSON, J. I. An examination of local versus nonlocal aspects of a tke-based boundary layer scheme in clear convective conditions. Journal of Applied Meteorology, v. 38, p. 1499-1518, 1999.

DELAGE, Y. A numerical study of the nocturnal atmospheric boundary layer. Quarterly Journal of the Royal Meteorological Society, v. 100, p. 251-265, 1974.

DELAGE, Y. Parameterising sub-grid scale vertical transport in atmospheric models under statically stable conditions. Boundary-Layer Meteorology, v. 82, p. 23-48, 1997.

LOUIS, J. F. A parametric model of vertical eddy fluxes in the atmosphere. BoundaryLayer Meteorology, v. 17, p. 165-179, 1979.

MCNIDER, R. T., et al.. Predictability of the stable atmospheric boundary layer. Journal of the Atmospheric Sciences, v. 52, p. 1602-1614, 1995.

van de WIEL, B. J. H.; RONDA, R. J.; MOENE, A. F.; BRUIN, H. A. R. D.; HOLTSLAG, A. A. M. Intermittent turbulence and oscillations in the stable boundary layer over land. part i: A bulk model. Journal of the Atmospheric Sciences, v. 59, p. 942-958, 2002. 